\title{
CLOSED-LOOP CONTROL OF A PARALLEL-PLATE MICROACTUATOR BEYOND THE PULL-IN LIMIT
}

\author{
Michael S.-C. Lu* and Gary K. Fedder ${ }^{* \dagger}$ \\ *Department of Electrical and Computer Engineering and ${ }^{\dagger}$ The Robotics Institute \\ Carnegie Mellon University, Pittsburgh, PA 15213-3890
}

\begin{abstract}
In this paper, we present a controller design for servoing the position of a parallel-plate electrostatic microactuator beyond its open-loop instability point. Controller design considers nonlinearities from both the parallel-plate actuator and the parallel-plate position sensor, to ensure robust stability within the feedback loop. Desired transient response is achieved by a pre-filter added in front of the feedback loop to shape the input command. The microactuator is characterized by static and dynamic measurements, with a spring constant of $0.17 \mathrm{~N} / \mathrm{m}$, mechanical resonant frequency of $12.4 \mathrm{kHz}$, and effective damping ratio from 0.55 to 0.35 for gaps between 2.3 to $2.65 \mu \mathrm{m}$. The minimum input-referred noise capacitance change is measured at a gap of $5.5 \mu \mathrm{m}$, corresponding to a minimum input-referred noise displacement of $0.2 \mathrm{~nm} / \sqrt{\mathrm{Hz}}$. Results of the servo test show excellent agreement with design specifications (Rise time $<2 \mathrm{~ms}$, overshoot $=0$, and settling time $<5 \mathrm{~ms}$ ) for the intended application as a magnetic probe tip actuator for data storage. Actuator displacement servoed as far as $55 \%$ of the gap is measured, which surpasses the static pull-in limit of one-third of the gap.
\end{abstract}

\section{INTRODUCTION}

For many years a well-known yet challenging problem for MEMS researchers interested in electrostatic actuation has been to extend the travel range of parallel-plate type electrostatic microactuators beyond the pull-in instability of one-third of the gap. Several open-loop methods have been proposed, including curved electrode design [2], "leveraged bending" method [3], and actuator redesign by adding a series feedback capacitance [4][5] to actuators. Those methods achieve large displacement at the cost of higher actuation voltage. Additional drawbacks of the series capacitor approach include: (1) actuator displacement is limited by parasitic capacitances, and (2) undesirable charge accumulated at the floating highimpedance node between actuated and series capacitances requires constant reset by a switch. Switch turn-off leads to charge injection into the actuated capacitor, which can be destabilizing if actuated capacitance is only several femtofarads. Successful measurements showing displacement as large as $88 \%$ and $60 \%$ of initial gap have been reported by [3] and [4], respectively. For applications which require accurate position servo under plant uncertainties and disturbances, closed-loop feedback has been analyzed [6]. Yet no measurement of this approach has been reported to the author's knowledge.

Research work presented in this paper originates from development of a probe-based magnetic micro disk drive [1] in Carnegie Mellon University. In the envisioned system, arrays of tip actuators are fabricated by a conventional CMOS process with integrated circuits for feedback control and data channels. By the parallel-plate electrostatic servo, actuators are controlled in parallel to the magnetic media atop for data read and write. Each actuator is nonlinear, and becomes unstable as it reaches the pull-in point and beyond. We select design of a linear time-invariant (LTI) controller over a nonlinear or a linear time-varying (LTV) controller for its easier imple- mentation by analog circuits. By linearization, stability analysis is performed in the frequency domain in order to maximize the phase and gain margins.

\section{BLOCK DIAGRAM}

The feedback system block diagram is illustrated in Figure 1, which has two transfer functions to be designed. One is the controller, $\mathrm{C}(\mathrm{s})$, for achieving robust stability within the loop, and the other is the pre-filter, $\mathrm{F}(\mathrm{s})$, which shapes the input command for the feedback loop to achieve desired output performance. The controlled plant consists of the parallel-plate electrostatic actuator and the parallel-plate capacitive position sensor.

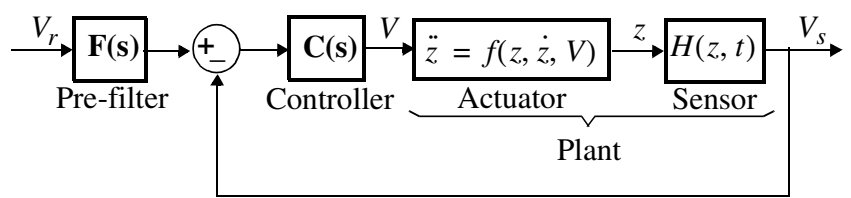

Figure 1. Two degree-of-freedom feedback system configuration.

\section{DEVICE FABRICATION}

Devices are fabricated by the AMS (Austria Micro System) $0.5 \mu \mathrm{m}$ three-metal-two-poly CMOS process, followed by postCMOS micromachining steps described in [8]. First, an anisotropic reactive-ion oxide etch (RIE) with $\mathrm{CHF}_{3} / \mathrm{O}_{2}$ plasma defines the structural sidewalls with the top metal layer used as an etch-resistant mask. The etch rate is $424 \AA / \mathrm{min}$. Next, an deep reactive-ion silicon etch is performed in an inductively-coupled-plasma etcher at an etch rate of $2.9 \mu \mathrm{m} / \mathrm{min}$. Finally microstructures are released from the substrate by an isotropic silicon etch at $1 \mu \mathrm{m} / \mathrm{min}$. The process flow is illustrated in Figure 2.

(a)

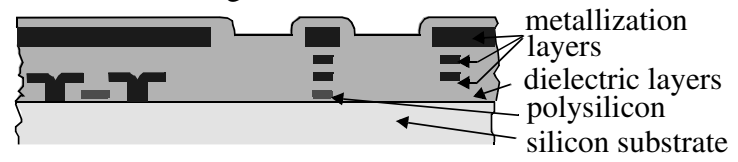

(b)

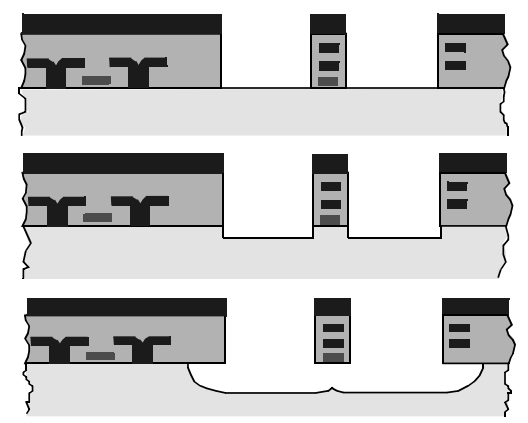

Figure 2. Cross section of the CMOS-MEMS process flow. (a). After CMOS processing. (b). After anisotropic dielectric reactiveion etch for definition of structural sidewalls. (c). After anisotropic silicon etch. (d). After isotropic silicon etch for structural release. 


\section{PLANT}

The dynamic equation of a conventional parallel-plate microactuator is described by

$$
m \ddot{z}+b \dot{z}+k z=\frac{\varepsilon_{o} A V^{2}}{2(g-z)^{2}}
$$

where $m$ is the mass, $b$ is the squeeze-film damping coefficient, $k$ is the spring constant, $\varepsilon_{o}$ is the permittivity of free space, $A$ is the parallel-plate area, and $V$ is the applied voltage. The gap, $g$, and displacement, $z$, are defined at the center of the actuated capacitance. A released microactuator is illustrated in Figure 3. It has two anchored springs, which carry actuation and sensing signals individually to avoid feedthrough. An external electrode is placed on top (not shown in the graph) of the actuator to establish the initial gap and the actuated and sensing capacitances. The actuation and sensing plates are mechanically connected, but electrically isolated from each other.

A schematic representation of the entire feedback system is illustrated in Figure 4. The position sensing is achieved by a singleended capacitive bridge with modulation/demodulation, followed by a low-pass filter to remove the $1 \mathrm{x}$ and $2 \mathrm{x}$ carrier frequency terms. The sensed output after the low-pass filter is

$$
V_{s}(z)=A_{v} A_{d} V_{m}\left(\frac{C_{s 1}(z)+C_{s 2}(z)}{C_{s 1}(z)+C_{s 2}(z)+C_{i}}-\frac{C_{s 1 o}+C_{s 2 o}}{C_{s 1 o}+C_{s 2 o}+C_{i}}\right)
$$

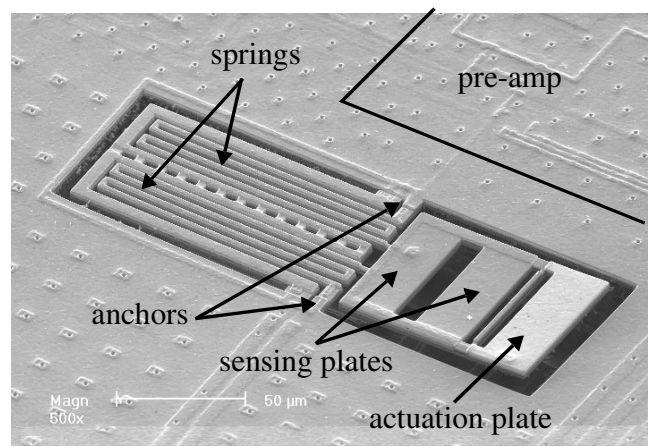

Figure 3. SEM of the released microactuator fabricated by CMOS-MEMS process.

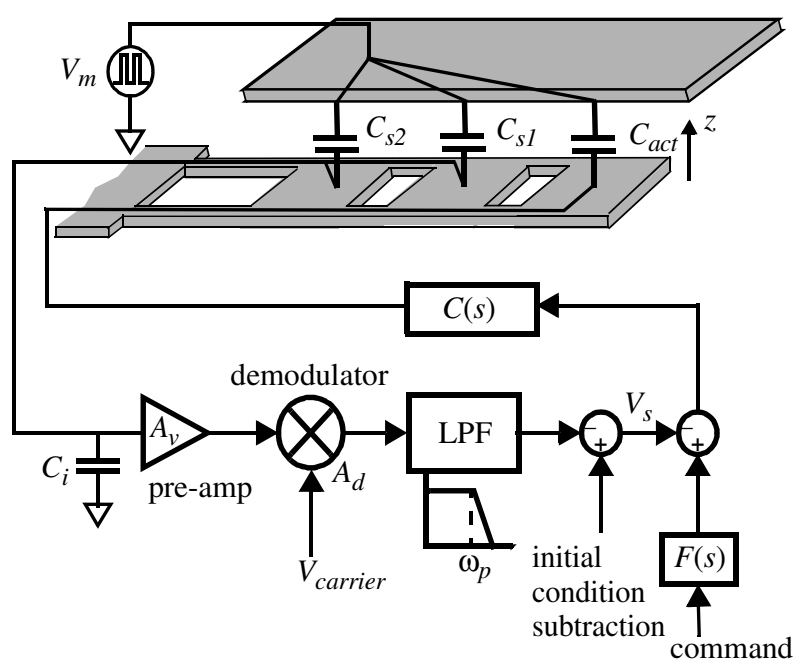

Figure 4. Schematic representation of the feedback control system. where $A_{v}$ is the pre-amp gain, $A_{d}$ is the demodulator gain, $C_{i}$ is the pre-amp input capacitance, and $C_{s 1 o}, C_{s 2 o}$ are the initial sensing capacitances. The displacements of the sensing plates are obtained by finite-element simulations [10] with given actuation plate displacements $z$ to account for the cantilever rotation. Substituting the displacements into the sensing capacitances gives

$$
C_{s 1}(z)=\varepsilon_{o} A /(g-0.84 z), C_{s 2}(z)=\varepsilon_{o} A /(g-0.67 z)
$$

The capacitive sensor gain at an operating point $z=Z_{o}$ is defined by differentiation of the sensed output with respect to the displacement. By including the dominant pole $\omega_{p}$ from the low-pass filter, the sensor transfer function at $z=Z_{o}$ is

$$
\left.H(s)\right|_{Z_{o}}=\frac{\left(d V_{s} / d z\right)_{Z_{o}}}{1+s / \omega_{p}}=\frac{G\left(Z_{o}\right)}{1+s / \omega_{p}}
$$

Plant parameters used for controller design are: $m=3 \times 10^{-11} \mathrm{~kg}, k=0.17 \mathrm{~N} / \mathrm{m}, A_{v}=10, A_{d}=5, A=1600 \mu \mathrm{m}^{2}$, $g=3 \mu \mathrm{m}$, and $\omega_{p}=120 \mathrm{kHz}$. The squeeze-film damping coefficient $b$ is computed between $1.8 \times 10^{-6}$ and $1.1 \times 10^{-3} \mathrm{~N} \cdot \mathrm{s} / \mathrm{m}$ at gaps between $3 \mu \mathrm{m}$ and $0.2 \mu \mathrm{m}$ by simulations [7].

\section{CONTROLLER DESIGN}

Consider a small variation of $\Delta z$ and $\Delta v$ around the operating point $\left(Z_{o}, V_{o}\right)$, the linearized actuator dynamics is derived by expanding the electrostatic force in the Taylor's series with the higher-order terms omitted:

$$
m \Delta \ddot{z}+b \Delta \dot{z}+\left(k-\left.\frac{\partial F_{e}}{\partial z}\right|_{z_{o}, V_{o}}\right) \Delta z=\left.\frac{\partial F_{e}}{\partial V}\right|_{Z_{o}, V_{o}} \Delta v
$$

where

$$
\left.\frac{\partial F_{e}}{\partial V}\right|_{Z_{o}, V_{o}}=\frac{2 k Z_{o}}{V_{o}}
$$

and

$$
\left.\frac{\partial F_{e}}{\partial z}\right|_{Z_{o}, V_{o}}=\left(\frac{2 \alpha}{1-\alpha}\right) k
$$

where $\alpha=Z_{o} / g$. Combining the transfer function of the linearized actuator and the capacitive sensor in (4) gives the linearized plant

$$
P(s)=\frac{G\left(Z_{o}\right) \cdot\left(\frac{2 k Z_{o}}{V_{o}}\right)}{\left(\frac{s}{\omega_{p}}+1\right)\left(m s^{2}+b s+\left(\frac{1-3 \alpha}{1-\alpha}\right) k\right)}
$$

Rewriting (8) using the resonant frequency $\omega_{n}=\sqrt{k / m}$ and the damping ratio $\xi=b /(2 \sqrt{\mathrm{km}})$, and multiplying the plant with a proportional-gain controller $C(s)=K_{p}$ gives the open-loop transfer function

$$
L(s)=\frac{K}{\left(\frac{s}{\omega_{p}}+1\right)\left(s^{2}+2 \xi_{e} \omega_{e} s-\omega_{e}^{2}\right)}
$$

where

$$
K=K_{p} G\left(Z_{o}\right)\left(\frac{2 Z_{o}}{V_{o}}\right) \omega_{n}^{2}
$$

and the effective resonant frequency and the effective damping ratio due to the spring-softening effect are 


$$
\omega_{e}=\sqrt{\frac{3 \alpha-1}{1-\alpha}} \omega_{n}, \quad \xi_{e}=\sqrt{\frac{1-\alpha}{3 \alpha-1}} \xi
$$

The magnitude and phase of $L(j \omega)$ are given by

$$
|L(j \omega)|=\frac{K}{\sqrt{1+\omega^{2} / \omega_{p}^{2}} \sqrt{\left(\omega^{2}+\omega_{p}^{2}\right)^{2}+\left(2 \xi_{e} \omega_{e} \omega\right)^{2}}}
$$

and

$$
\angle L(j \omega)=-\pi+\tan ^{-1}\left(\frac{2 \xi_{e} \omega_{e} \omega}{\omega^{2}+\omega_{e}^{2}}\right)-\tan ^{-1}\left(\frac{\omega}{\omega_{p}}\right)
$$

Substituting the phase margin $\phi$ and the unity-gain frequency $\omega=\omega_{\phi}$ into (13) gives

$$
\frac{2 \xi_{e} \omega_{e} \omega_{\phi}}{\omega_{\phi}^{2}+\omega_{e}^{2}}=\frac{\tan (\phi)-\frac{\omega_{\phi}}{\omega_{p}}}{1+\tan (\phi) \cdot \frac{\omega_{\phi}}{\omega_{p}}}
$$

Equating $\angle L(j \omega)$ to $-\pi$ at the upper gain-margin frequency $\omega=\omega_{g}$ yields

$$
\omega_{g}=\sqrt{\omega_{e}\left(2 \xi_{e} \omega_{p}-\omega_{e}\right)}
$$

Then substituting (15) into (12) gives the upper gain margin

$$
G_{H}=\left(\frac{K}{2 \xi_{e} \omega_{p} \omega_{e}\left(1+2 \xi_{e} \omega_{e} / \omega_{p}-\omega_{e}^{2} / \omega_{p}^{2}\right)}\right)^{-1}
$$

Equating $|L(j \omega)|$ to one at $\omega=\omega_{\phi}$ yields

$$
K=\frac{\sqrt{\omega_{\phi}^{2}+\omega_{p}^{2}} \sqrt{\left(\omega_{\phi}^{2}+\omega_{e}^{2}\right)^{2}+\left(2 \xi_{e} \omega_{e} \omega_{\phi}\right)^{2}}}{\omega_{p}}
$$

By replacing $\omega=0$ and (17) into (12), the lower gain margin is given by

$$
G_{L}=\sqrt{\left(\frac{\omega_{\phi}}{\omega_{p}}\right)^{2}+1} \sqrt{\left(\frac{\omega_{\phi}^{2}}{\omega_{e}^{2}}+1\right)^{2}+\left(\frac{2 \xi_{e} \omega_{\phi}}{\omega_{e}}\right)^{2}}
$$

To find the maximum phase margin, $\angle L(j \omega)$ is differentiated with respect to $\omega$ at $\omega=\omega_{\phi}$. The resultant unity-gain frequency is the solution of the following quartic equation:

$$
\begin{aligned}
& \left(-\omega_{p}-2 \xi_{e} \omega_{e}\right) \omega_{\phi}^{4}+\left(-2 \xi_{e} \omega_{p}^{2} \omega_{e}-2 \omega_{p} \omega_{e}^{2}-4 \xi_{e}^{2} \omega_{p} \omega_{e}^{2}\right. \\
& \left.+2 \xi_{e} \omega_{e}^{3}\right) \omega_{\phi}^{2}+\left(2 \xi_{e} \omega_{p}^{2} \omega_{e}^{3}-\omega_{p} \omega_{e}^{4}\right)=0
\end{aligned}
$$

Given values of $\omega_{p} / \omega_{\mathrm{e}}$ and $\xi_{e}$ according to the ratio of $Z_{o} / g$, $\omega_{\phi}$ is first solved from (19). Then maximum phase margin, upper and lower gain margins and the controller $K_{p}$ are computed by (14), (16), (18), and (10). Calculated maximum phase margins with respect to the actuator displacement are plotted in Figure 5. Since a LTI controller will be implemented, selected controller should give reasonable phase and gain margins at each displacement. The final design of the controller and pre-filter are:

$$
C(s)=17 \text { and } F(s)=\frac{1}{1+s / 1800}
$$

with the resultant phase margins shown in Figure 5.

\section{CHARACTERIZATION}

For static measurement, a d.c. voltage is incrementally applied to the actuator, and an a.c. modulation voltage is applied to the top electrode for capacitive sensing. The spring constant of the actuator is extracted from the displacement-voltage characteristic in

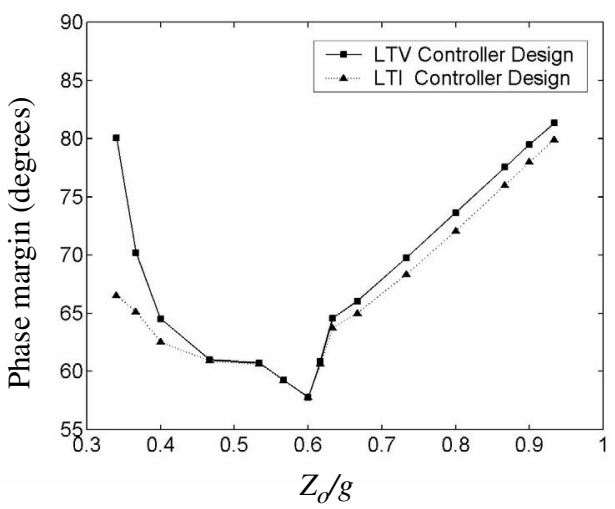

Figure 5. The maximum phase margin corresponding to the actuator displacement.

Figure 6 at $0.17 \mathrm{~N} / \mathrm{m}$, and the input capacitance $C_{i}$ is computed at $333 \mathrm{fF}$ from measurements.

The actuator resonant frequency is measured electronically by the Agilent 4395A spectrum/network analyzer, which gathers readout signal from the demodulator output to construct the actuator frequency response. The resonant frequency decreases as a result of the spring-softening effect from the increasing d.c. bias. Both experimental and NODAS simulation results [9] are shown in Figure 7, in which the actuator resonant frequency is found at $12.4 \mathrm{kHz}$. From the resonant peak of the measured frequency response, effective damping ratio is calculated between 0.55 to 0.35 for gaps between 2.3 to $2.65 \mu \mathrm{m}$.

Measured frequency response of the sensing pre-amp has a closed-loop gain of ten and the corner frequency at $6.3 \mathrm{MHz}$. The minimum input-referred noise voltage is measured at $1.1 \mu \mathrm{V} / \sqrt{ } \mathrm{Hz}$,

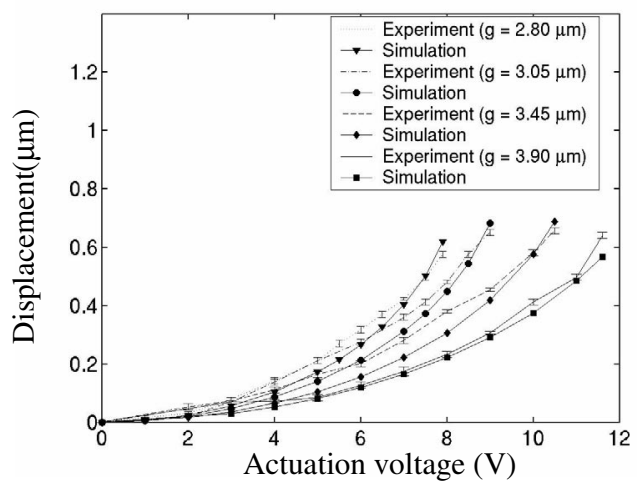

Figure 6. The static displacement-voltage characteristic measures the actuator spring constant at $0.17 \mathrm{~N} / \mathrm{m}$.

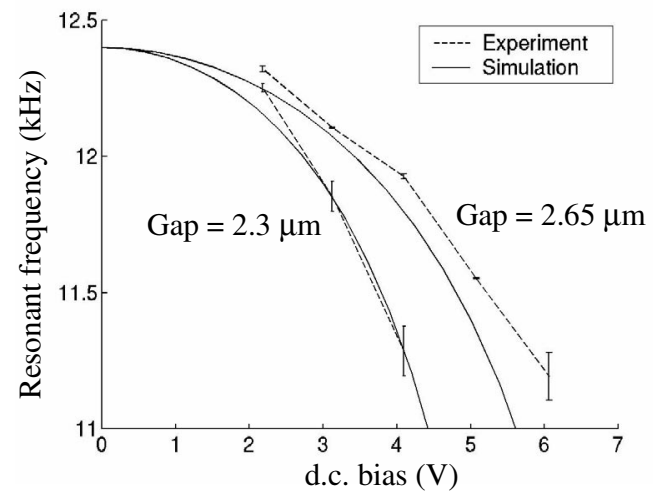

Figure 7. Measured resonant frequency and its shift due to the electrostatic spring-softening effect. 


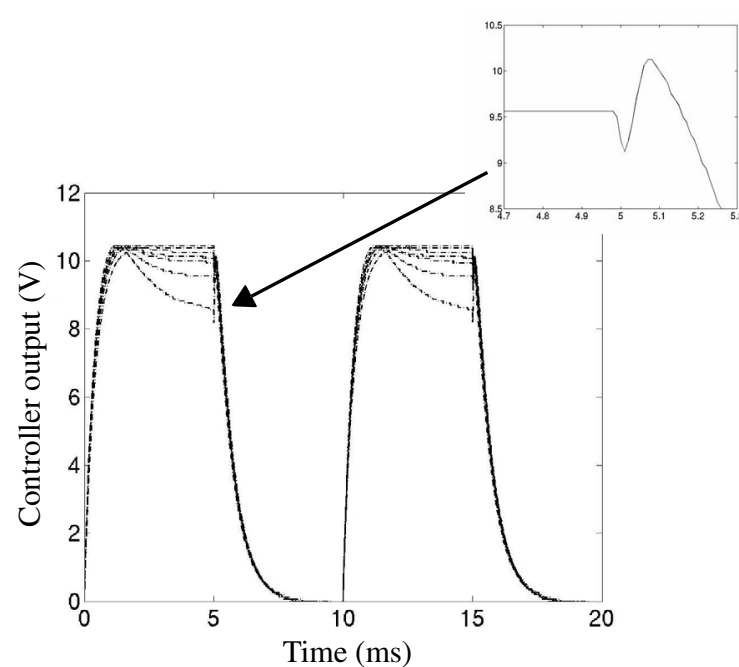

Figure 8. Measured controller output waveforms when displaced plate enters pull-in region and beyond. The inset illustrates its waveform when the input command turns from high to low.

which is equal to a minimum input-referred noise capacitance change and a minimum input-referred noise displacement of $0.25 \mathrm{aF} / \sqrt{ } \mathrm{Hz}$ and $0.2 \mathrm{~nm} / \sqrt{\mathrm{Hz}}$, respectively.

\section{CLOSED-LOOP POSITION SERVO}

The input command is a square waveform at $100 \mathrm{~Hz}$ with the minimum set at zero. A series of controller outputs is measured when the actuator moves across the pull-in instability and beyond, as shown in Figure 8. The decrease of controller output after the pull-in voltage $(10.44 \mathrm{~V})$ reduces the stored charge on the plate in order to stabilize the actuator in the unstable regime. As the input command changes from high to low, the controller output first decreases, and then increases as the actuator retracts back to the rest position to maintain stability, as shown by the inset in Figure 8 . The actuator displacements, as depicted in Figure 9, are extracted from the measured sensor output voltage waveforms. A maximum displacement of $55 \%$ the gap $(g=3.25 \mu \mathrm{m})$ is illustrated, with the rise time less than $2 \mathrm{~ms}$, the settling time less than $5 \mathrm{~ms}$, and no overshoot.

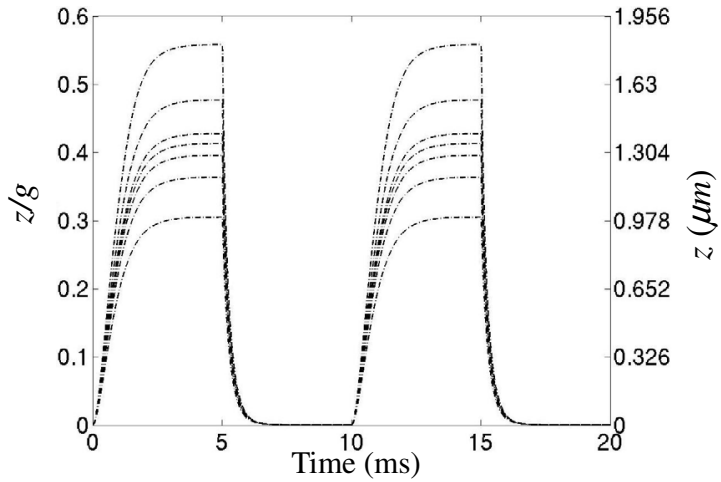

Figure 9. Transient response of the actuator displacement. $(g=$ $3.25 \mu \mathrm{m})$.

\section{DISCUSSION AND CONCLUSION}

Controller design and the closed-loop implementation for the parallel-plate capacitor servo are discussed. With a proportionalgain controller, nonlinearities of the actuator and capacitive sensor are considered. Actuator displacement servoed as far as $55 \%$ of the initial gap is demonstrated. The maximum servo range is limited due to the following reasons: (1) structural curl of the fabricated actuator results in tilted plates and thus reduced damping coefficient, which leads to decrease of the phase margin, and (2) the implemented loop bandwidth is limited by the use of low-pass filtering after the demodulator. Hence no phase-lead compensation can be attempted for the phase loss. From the controller design standpoint, use of a LTI controller facilitates the implementation but is less versatile than other alternatives such as a nonlinear controller and a LTV controller, in terms of bandwidth efficiency and attainable gain and phase margins. Those types of controllers remain interesting research topics for the future.

\section{ACKNOWLEDGEMENT}

This work is sponsored by the Defense Advanced Research Project Agency (DARPA) under agreement number F306029820239.

\section{REFERENCES}

1. L. R. Carley, J. A. Bain, G. K. Fedder, D. W. Greve, D. F. Guillou, M. Lu, T. Mukherjee, S. Santhanam, L. Abelmann, and S. Min, "Single-chip computers with microelectromechanical system-based magnetic memory", Journal of Applied Physics, vol. 87 , no. 9, pt. 1-3, (2000), pp. 6680-6685.

2. R. Legtenberg, J. Gilbert, S. D. Senturia, and M. Elwenspoek, "Electrostatic curved electrode actuators", J. of Microelectromechanical Systems, vol. 6, no.3, (1997), pp. 257-265.

3. E. S. Hung and S. D. Senturia, "Extending the travel range of analog-tuned electrostatic actuators", J. of Microelectromechanical Systems, vol. 8, no.4, (1999), pp. 497-505.

4. E. K. Chan and R. W. Dutton, "Electrostatic micromechanical actuator with extended range of travel", J. of Microelectromechanical Systems, vol. 9, no.3, (2000), pp. 321-328.

5. J. I. Seeger, B. E. Boser, "Dynamics and control of parallelplate actuators beyond the electrostatic instability", Proc. Transducers'99, Sendai, Japan, (1999), pp. 474-477.

6. P. B. Chu, K. S. Pister, "Analysis of closed-loop control of parallel-plate electrostatic microgrippers", in Proc. IEEE int. conf. Robotics and Automation, San Diego, CA, (1994), pp. 820-825.

7. S. Vemuri, G. K. Fedder, and T. Mukherjee, "Low-order squeeze film model of simulation of MEMS devices," International conference on Modeling and Simulation of Microsystems, San Diego, CA, pp. 205-208, 2000.

8. G. K. Fedder, S. Santhanam, M. L. Reed, S. C. Eagle, D. F. Guillou, M. Lu, and L. R. Carley, "Laminated high-aspect-ratio microstructures in a conventional CMOS process", Sensors and Actuators A (Physical), vol. A57, no. 2, (1997), pp. 103-110.

9. G. K. Fedder, Q. Jing, "A hierarchical circuit-level design methodology for microelectromechanical systems," IEEE Trans. on Circuits and Systems II: Analog and Digital Signal Processing, vol.46, no.10, (1999), pp. 1309-1315.

10. ABAQUS manual, version 6.2, Hibbitt, Karlsson \& Sorensen, Inc., (2001). 